\title{
Can persistent organic pollutants explain the association between serum $\gamma$-glutamyltransferase and type 2 diabetes?
}

\author{
D.-H. Lee • M. W. Steffes • D. R. Jacobs Jr.
}

Received: 1 August 2007 / Accepted: 12 November 2007 / Published online: 11 December 2007

(C) Springer-Verlag 2007

\begin{abstract}
The results of several epidemiological studies of serum $\gamma$-glutamyltransferase (GGT) led us to hypothesise that associations of GGT within its normal range with type 2 diabetes may reflect detrimental effects of xenobiotics found in the environment, such as persistent organic pollutants (POPs). Epidemiological observations showed that serum GGT activity within its normal range strongly predicted future type 2 diabetes; the predictability of diabetes from obesity was low with GGT at the low end of the normal range; and GGT showed a positive association with known markers of oxidative stress or inflammation. Experimental findings on cellular GGT suggest that serum GGT levels within the normal range may reflect oxidative stress related
\end{abstract}

\section{D.-H. Lee}

Department of Preventive Medicine and Health Promotion

Research Center, School of Medicine,

Kyungpook National University,

Daegu, South Korea

M. W. Steffes

Department of Laboratory Medicine and Pathology,

University of Minnesota,

Minneapolis, MN, USA

D. R. Jacobs Jr.

Division of Epidemiology, School of Public Health, University of Minnesota,

Minneapolis, MN, USA

D. R. Jacobs Jr.

Department of Nutrition, University of Oslo,

Oslo, Norway

D.-H. Lee $(\bowtie)$

Department of Preventive Medicine, School of Medicine,

Kyungpook University,

101 Dongin-dong, Jung-gu,

Daegu 700-422, South Korea

e-mail: lee_dh@knu.ac.kr to the re-synthesis of intracellular glutathione; however, this interpretation is not completely satisfying because, in its role of regenerating intracellular glutathione, GGT activity should be antioxidative. Alternatively, serum GGT activity may reflect amounts of glutathione conjugates formed during the metabolism of xenobiotics. Accordingly, we postulate a two-part hypothesis: that the association of serum GGT with type 2 diabetes reflects exposure to POPs, as these substances, which have a very long half-life, may influence diabetes risk by residing in adipose tissue as endocrine disruptors; and that POPs or similar substances may interact with obesity to cause type 2 diabetes. Supporting this hypothesis, cross-sectional investigation of background exposure to POPs in the National Health and Nutrition Examination Survey showed relationships similar to those observed for GGT, including a powerful association with prevalent diabetes and no association between obesity and diabetes for very low POP concentrations. Our hypothesis can be tested in both prospective studies and toxicological studies.

Keywords Environmental pollutants .

$\gamma$-Glutamyltransferase - Obesity .

Persistent organic pollutants · Type 2 diabetes

\begin{tabular}{|c|c|}
\hline Abbreviations & \\
\hline CARDIA & $\begin{array}{l}\text { Coronary Artery Risk Development } \\
\text { in Young Adults }\end{array}$ \\
\hline FinMONICA & $\begin{array}{l}\text { Finland Multinational Monitoring } \\
\text { of Trends and Determinants in } \\
\text { Cardiovascular Disease }\end{array}$ \\
\hline GGT & $\gamma$-glutamyltransferase \\
\hline GSH & glutathione \\
\hline NHANES & National Health and Nutrition \\
\hline OC pesticides & $\begin{array}{l}\text { Examination Survey } \\
\text { organochlorine pesticides }\end{array}$ \\
\hline
\end{tabular}


POPs persistent organic pollutants

ROS

reactive oxygen species

\section{$\gamma$-Glutamyltransferase and diabetes mellitus}

Our hypothesis and observations regarding persistent organic pollutants (POPs) and type 2 diabetes arose from interpretations of several studies of serum $\gamma$-glutamyltransferase (GGT). Serum GGT activity within the normal range predicted type 2 diabetes in Korean men [1]. Furthermore, the association between serum GGT and type 2 diabetes was stronger among the obese, and obesity did not predict the risk of type 2 diabetes among participants with GGT levels at the low end of the normal range [1]. A similar pattern of associations was observed in datasets from the Coronary Artery Risk Development in Young Adults (CARDIA) study [2], the Finland Multinational Monitoring of Trends and Determinants in Cardiovascular Disease (FinMONICA) study [3], the National Health and Nutrition Examination Survey (NHANES) [4], and in German women, but not men [5], leading us to search for new interpretations of serum GGT.

Cellular GGT, found on the plasma membrane in most tissues [6], metabolises extracellular glutathione (GSH), the main intracellular antioxidant, allowing precursor amino acids to be reutilised for intracellular GSH re-synthesis [7]a process known as the ' $\gamma$-glutamyl cycle' (Fig. 1, left of the dotted line) [8]. Cellular GGT may also be involved in the generation of reactive oxygen species (ROS) in the presence of transition metals [9].

This information on cellular GGT led us to hypothesise that serum GGT levels within the normal range reflect oxidative stress [10]. Supporting this hypothesis, serum GGT is inversely associated with serum antioxidants [11] and positively associated with $\mathrm{F}_{2}$-isoprostanes and C-reactive protein [2].

However, this interpretation of serum GGT presented a dilemma. In terms of the $\gamma$-glutamyl cycle, an increase in GGT should the facilitate regeneration of intracellular GSH, eventually leading to increased levels, even though the initial increase may be a compensatory response to the depletion of GSH as a result of oxidative stress. Thus, an increase in GGT should theoretically lead to a decrease in oxidative stress and consequent pathological changes. However, prospective cohort studies have consistently reported that a slight increase in serum GGT activity precedes an increase in type 2 diabetes risk.

Some researchers interpreted that serum GGT predicts type 2 diabetes because it acts as a pro-oxidant or a marker of hepatic steatosis [12-14]. These mechanisms may partly explain some of the associations between GGT and type 2 diabetes, especially those observed at relatively high levels of serum GGT activity. However, it is important that even a slight increase in serum GGT activity at the low end of the normal range, where hepatic steatosis is unlikely, increased the risk of type 2 diabetes [1-3].
Fig. 1 The section to the right of the dotted line shows the role of GGT in the metabolism of xenobiotics. Cellular GGT is necessary for the metabolism of xenobiotics conjugated with GSH. The section to the left of the dotted line shows the $\gamma$ glutamyl cycle. Cellular GGT is an indispensable enzyme in the metabolism of extracellular GSH. In this process, GGT releases glutamate (Glu) and the dipeptide cysteinylglycine (CG), which is subsequently cleaved into cysteine (Cys) and glycine (Gly) by plasma membrane dipeptidase (DP). CYP450, cytochrome $\mathrm{P} 450$

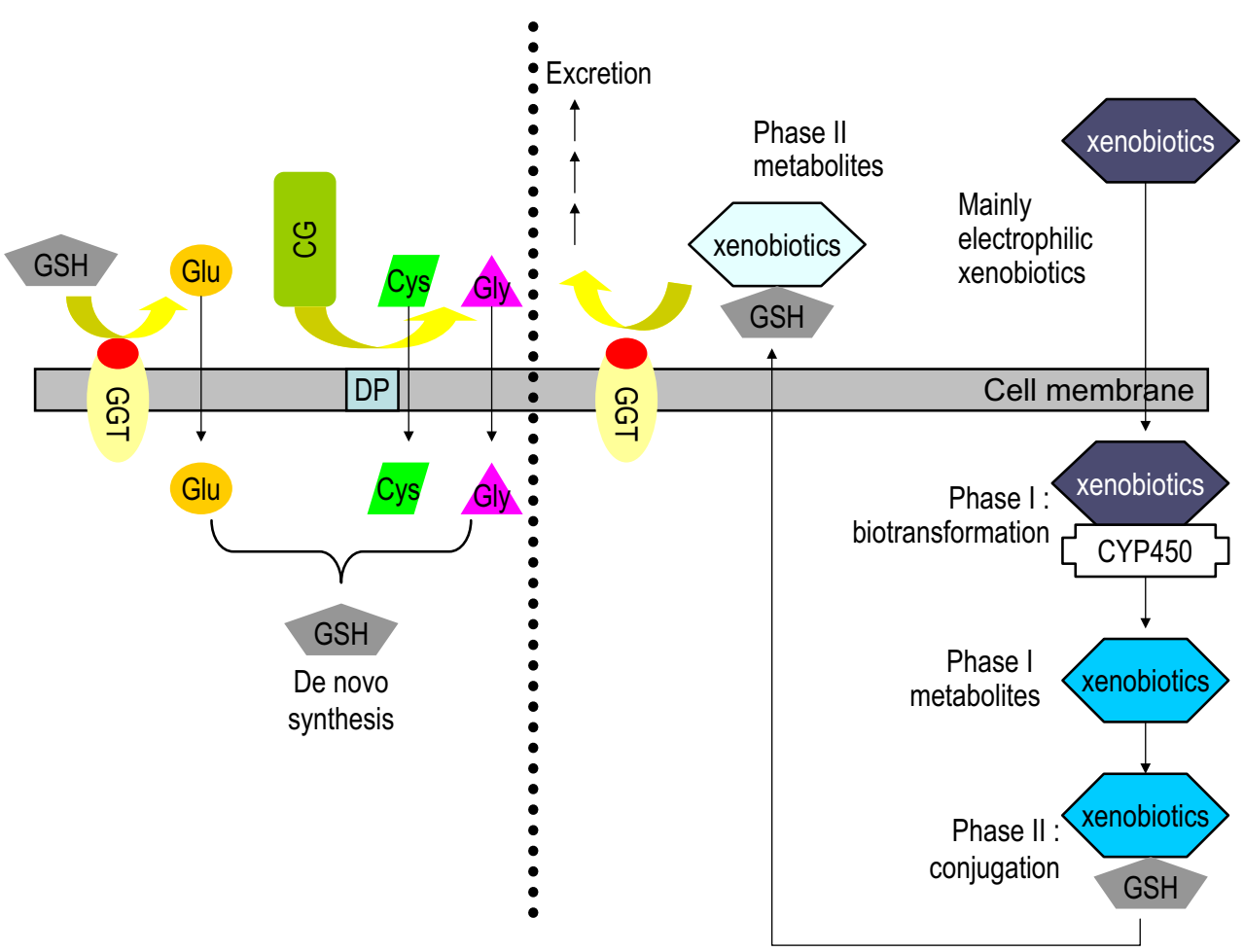




\section{Serum GGT activity as a cumulative exposure marker for various xenobiotics}

Although the aforementioned study on Korean men [1] revealed much lower serum GGT activity than that observed in CARDIA and FinMONICA participants [2, 3], there was a striking secular trend in serum GGT [15]. After statistical adjustment, we concluded that the secular trend was not caused by changes in health behaviours or obesity, suggesting that other environmental factors play a role.

Another avenue of thought arose from the observation that serum GGT demonstrated dose-response relationships with blood concentrations of lead and urinary levels of cadmium [16]. This work brought into focus the important function of GSH as a conjugating ligand for the phase II reactions that occur during xenobiotic metabolism. Many xenobiotics are eliminated from cells by phase I biotransformation, followed by phase II conjugation to an anionic group, such as GSH, and transportation into the extracellular space [17]. The first necessary step for further metabolism of GSH conjugates is to break $\gamma$-carboxyl linkage of GSH by cellular GGT (Fig. 1, right of the dotted line) [18]. Thus, GGT activity may increase with an increased exposure to xenobiotics, including environmental pollutants, which require conjugation with GSH [17]. Our updated viewpoint on serum GGT as a marker of exposure to xenobiotics is a more comprehensive interpretation, which includes our earlier proposal that serum GGT reflects oxidative stress. Exposure of cells to xenobiotics directly increases the production of ROS [19]. ROS are also conjugated with GSH [17], and consumption of GSH by conjugation with xenobiotics is related to the depletion of intracellular GSH [17]. Based on these propositions, we hypothesised that the associations between serum GGT activity and type 2 diabetes might be explained by exposure to environmental pollutants.

\section{Serum GGT activity as a marker of exposure to POPs}

For our hypothesis to be true, the environmental pollutants involved have to satisfy several conditions. First, human exposure to the presumed xenobiotics should be through food consumption, most likely meat. For example, the Korean secular trend of increasing serum GGT activity was similarly observed among all subgroups stratified by various factors, such as age, sex, smoking, alcohol drinking, obesity or job category [15], suggesting some common environmental exposures, such as food. Indeed, serum GGT was positively associated with meat intake in the CARDIA dataset [20]. Second, the xenobiotics should be associated with adipose tissue, because serum GGT activity is strongly associated with obesity. Considering the importance of adipose tissue in the pathogenesis of type 2 diabetes, pollutants stored in adipose tissue could be important. Third, the xenobiotics should be metabolised by GSH conjugation.

Following this logic, POPs, endocrine disruptors stored in adipose tissue, represented the most plausible candidate. POPs include hundreds of different chemical compounds with common properties, such as long-term persistence in the environment and bioaccumulation through the food chain. POPs are detectable in virtually everyone, with exposure occurring through fatty animal food in particular [21]. Some POPs are conjugated to GSH for their metabolism [22-24], and exposure to high amounts of certain POPs in occupational or accidental settings increase serum GGT activity [25, 26].

We tested this hypothesis in the NHANES dataset and found graded associations between serum concentrations of POPs and serum GGT [27]. We also found strong doseresponse relationships between POPs and the prevalence of type 2 diabetes [28]. Parallel to the interactions of obesity and diabetes with serum GGT activity, the association between POPs and type 2 diabetes was stronger among obese persons, but type 2 diabetes was nearly absent, irrespective of obesity when POPs concentrations were very low [28]. This observation led us to hypothesise that POPs stored in adipose tissue might be more critical than obesity itself to understanding the pathogenesis of type 2 diabetes.

The studies described above focused on type 2 diabetes risk, reflecting our original hypothesis. However, serum GGT activity within its normal range has also been shown to prospectively predict other clinical outcomes [29-33]. Similarly, in the NHANES dataset, serum POP concentrations were positively associated with the prevalence of the metabolic syndrome, insulin resistance, hypertension, and cardiovascular diseases [34, 36].

Mitochondrial dysfunction has recently emerged as a mechanism unifying the pathogenesis of insulin resistance and type 2 diabetes [37]. Interestingly, it has long been reported that POPs can decrease mitochondrial oxidative capacity in various organs [38, 39]. Even though POPs are mainly stored in adipose tissue, physiological fatty acid release from adipose tissue between meals may be accompanied by some release of POPs, and they may keep redistributing to various organs, such as muscle, liver, or pancreas, even under normal physiological control of adipose tissue [40]. Furthermore, the lipolytic potential of some POPs may disturb normal adipose tissue metabolism and lead to an excessive release of POPs [41]. Thus, continuous chronic exposure to POPs may diminish mitochondrial function in various organs, eventually leading to insulin resistance and type 2 diabetes.

\section{Critiques of the hypothesis}

Counter to our hypothesis, type 2 diabetes is prevalent across countries with varying meat intakes. Although meat 
may be the putative source of POPs, through bioaccumulation in the food chain, in developed countries where POPs were banned decades ago, this may not be the case in developing countries, where some POPs are still widely used [42]. If local agriculture utilises organochloride pesticides, the subclass of POPs that showed the strongest associations with type 2 diabetes and insulin resistance [28, 35], fruits and vegetables could be the main source of POPs. Furthermore, POP content differs across meats, with fish usually containing higher concentrations of POPs than mammals [43]. Cooking method is another important factor affecting POP absorption [44]. Thus, ecological comparisons of meat intake by country or time may not be fully relevant to our hypothesis.

The fact that an association between obesity and type 2 diabetes was observed as early as the 19th century [45, 46] provides evidence against our hypothesis. Although most POPs are man-made in the 20th century, dioxins can be created in natural events such as forest fires or volcanic eruptions [47]; and POPs may not be the only substances that can interact with adipose tissue to cause type 2 diabetes. Our hypothesis largely concerns the epidemic of type 2 diabetes after the Second World War.

Further evidence that might counter our hypothesis subsumes declining body burden of POPs in developed countries [48], even as the epidemic of type 2 diabetes increases. However, POPs may have increased potency at a lower body burden in an obesity epidemic, because the toxicity of POPs appears to increase among obese persons [29]. Additionally, other POPs, such as brominated flame retardants and perfluorinated compounds, which are still widely used today, may be as important as the POPs we studied in the pathogenesis of type 2 diabetes [49]. Furthermore, the current generation may be experiencing cumulative epigenetic changes due to POPs [50], and these changes could predispose individuals to diabetes.

\section{Conclusion}

Starting with epidemiological studies of serum GGT, we formed a two-part hypothesis: the association of serum GGT with type 2 diabetes reflects exposure to POPs, and POPs interact with obesity to cause type 2 diabetes. Although various researchers have recently commented on the predictive power of serum GGT [17-19], none considered the possibility that serum GGT was a cumulative biomarker of environmental pollutants.

Furthermore, several lines of evidence suggest that if people had no or very low exposure to POPs, reflected by very low serum GGT activity, the probability of developing type 2 diabetes could be low, even among the obese. In this sense, POPs stored in adipose tissue may interact with this tissue to cause type 2 diabetes. Our hypothesis linking obesity, serum GGT, POPs and type 2 diabetes considers the interactions between all the predisposing factors, suggests
Fig. 2 A new hypothesis about the inter-relationships of serum GGT, POPs and type 2 diabetes. Serum GGT activity increases with increasing xenobiotic-GSH conjugation, including POPs. As endocrine disruptors, POPs can disturb glucose and lipid metabolism through mitochondrial dysfunction leading to an increase in the risk of type 2 diabetes. Obesity also contributes to type 2 diabetes by increasing the storage time of POPs in adipose tissue and enhancing toxicity of POPs. Some POPs may directly contribute to obesity. Epigenetic changes resulting from exposure to POPs over several generations may also be involved in the pathogenesis of type 2 diabetes. With genetic factors, the background exposure to POPs and their interactions with obesity can help to explain the recent epidemic of type 2 diabetes




an important role for the population-wide exposure to POPs (Fig. 2), and may help define the way in which obesity predisposes individuals to type 2 diabetes. Testing the hypothesis in prospective cohorts and in toxicological studies could help clarify whether and how specific POPs relate to type 2 diabetes.

Duality of interest The authors declare that there is no duality of interest associated with this manuscript.

\section{References}

1. Lee DH, Ha MH, Kim JH et al (2003) Gamma-glutamyltransferase and diabetes - a 4 year follow-up study. Diabetologia 46:359-364

2. Lee DH, Jacobs DR Jr, Gross M et al (2003) Gammaglutamyltransferase is a predictor of incident diabetes and hypertension: the Coronary Artery Risk Development in Young Adults (CARDIA) Study. Clin Chem 49:1358-1366

3. Lee DH, Silventoinen K, Jacobs DR Jr, Jousilahti P, Tuomileto J (2004) Gamma-glutamyltransferase, obesity, and the risk of type 2 diabetes: observational cohort study among 20,158 middle-aged men and women. J Clin Endocrinol Metab 89:5410-5414

4. Lim JS, Lee DH, Park JY, Jin SH, Jacobs DR Jr (2007) A strong interaction between serum gamma-glutamyltransferase and obesity on the risk of prevalent type 2 diabetes: results from the Third National Health and Nutrition Examination Survey. Clin Chem 53:1092-1098

5. Meisinger C, Lowel H, Heier M, Schneider A, Thorand B; KORA Study Group (2005) Serum gamma-glutamyltransferase and risk of type 2 diabetes mellitus in men and women from the general population. J Intern Med 258:527-535

6. Goldberg DM (1980) Structural, functional, and clinical aspects of gamma-glutamyltransferase. CRC Crit Rev Clin Lab Sci 12:1-58

7. Whitfield JB (2001) Gamma glutamyl transferase. Crit Rev Clin Lab Sci 38:263-355

8. Meister A (1974) Glutathione, metabolism and function via the gamma-glutamyl cycle. Life Sci 15:177-190

9. Stark AA, Zeiger E, Pagano DA (1993) Glutathione metabolism by gamma-glutamyltranspeptidase leads to lipid peroxidation: characterization of the system and relevance to hepatocarcinogenesis. Carcinogenesis 14:183-189

10. Lee DH, Blomhoff R, Jacobs DR Jr (2004) Is serum gamma glutamyltransferase a marker of oxidative stress? Free Radic Res 38:535-539

11. Lee DH, Gross MD, Jacobs DR Jr (2004) Association of serum carotenoids and tocopherols with gamma-glutamyltransferase: the Cardiovascular Risk Development in Young Adults (CARDIA) Study. Clin Chem 50:582-588

12. Grundy SM (2007) Gamma-glutamyl transferase: another biomarker for metabolic syndrome and cardiovascular risk. Arterioscler Thromb Vasc Biol 27:4-7

13. Whitfield JB (2007) Serum gamma-glutamyltransferase and risk of disease. Clin Chem 53:1-2

14. Emdin M, Pompella A, Paolicchi A (2005) Gamma-glutamyltransferase, atherosclerosis, and cardiovascular disease: triggering oxidative stress within the plaque. Circulation 112:2078-2080

15. Lee DH, Ha MH, Kam S et al (2006) A strong secular trend in serum gamma-glutamyltransferase from 1996 to 2003 among South Korean men. Am J Epidemiol 163:57-65
16. Lee DH, Lim JS, Song K, Boo Y, Jacobs DR Jr (2006) Graded associations of blood lead and urinary cadmium concentrations with oxidative-stress-related markers in the U.S. population: results from the third National Health and Nutrition Examination Survey. Environ Health Perspect 114:350-354

17. Strange RC, Spiteri MA, Ramachandran S, Fryer AA (2001) Glutathione- $S$-transferase family of enzymes. Mutat Res 482:21-26

18. Hinchman CA, Ballatori N (1994) Glutathione conjugation and conversion to mercapturic acids can occur as an intrahepatic process. J Toxicol Environ Health 41:387-409

19. Wells PG, Kim PM, Laposa RR, Nicol CJ, Parman T, Winn LM (1997) Oxidative damage in chemical teratogenesis. Mutat Res 396:65-78

20. Lee DH, Steffen LM, Jacobs DR Jr (2004) Association between serum gamma-glutamyltransferase and dietary factors: the Coronary Artery Risk Development in Young Adults (CARDIA) Study. Am J Clin Nutr 79:600-605

21. Abelsohn A, Gibson BL, Sanborn MD, Weir E (2002) Identifying and managing adverse environmental health effects: 5. Persistent organic pollutants. CMAJ 166:1549-1554

22. Schrenk D (1998) Impact of dioxin-type induction of drugmetabolizing enzymes on the metabolism of endo- and xenobiotics. Biochem Pharmacol 55:1155-1162

23. Mio T, Sumino K (1985) Mechanism of biosynthesis of methylsulfones from PCBs and related compounds. Environ Health Perspect 59:129-135

24. Anguiano OL, Caballero de Castro A, Pechen de D'Angelo AM (2001) The role of glutathion conjugation in the regulation of early toad embryos' tolerance to pesticides. Comp Biochem Physiol C Toxicol Pharmacol 128:35-43

25. Calvert GM, Hornung RW, Sweeney MH, Fingerhut MA, Halperin WE (1992) Hepatic and gastrointestinal effects in an occupational cohort exposed to 2,3,7,8-tetrachlorodibenzo-para-dioxin. JAMA 267:2209-2214

26. Mocarelli P, Marocchi A, Brambilla P, Gerthoux P, Young DS, Mantel N (1986) Clinical laboratory manifestations of exposure to dioxin in children. A six-year study of the effects of an environmental disaster near Seveso, Italy. JAMA 256:2687-2695

27. Lee DH, Jacobs DR Jr (2006) Association between serum concentrations of persistent organic pollutants and gamma glutamyltransferase: results from the National Health and Examination Survey 1999-2002. Clin Chem 52:1825-1827

28. Lee DH, Lee IK, Song K et al (2006) A strong dose-response relation between serum concentrations of persistent organic pollutants and diabetes: results from the National Health and Examination Survey 1999-2002. Diabetes Care 29:1638-1644

29. Ruttmann E, Brant LJ, Concin H, Diem G, Rapp K, Ulmer H (2005) Gamma-glutamyltransferase as a risk factor for cardiovascular disease mortality: an epidemiological investigation in a cohort of 163,944 Austrian adults. Circulation 112:2130-2137

30. Miura K, Nakagawa H, Nakamura H et al (1994) Serum gammaglutamyl transferase level in predicting hypertension among male drinkers. J Hum Hypertens 8:445-449

31. Jousilahti P, Rastenyte D, Tuomilehto J (2000) Serum gammaglutamyl transferase, self-reported alcohol drinking, and the risk of stroke. Stroke 31:1851-1855

32. Ryu S, Chang Y, Kim DI, Kim WS, Suh BS (2007) Gammaglutamyltransferase as a predictor of chronic kidney disease in nonhypertensive and nondiabetic Korean men. Clin Chem 53:71-77

33. Lee DS, Evans JC, Robins SJ et al (2007) Gamma glutamyl transferase and metabolic syndrome, cardiovascular disease, and mortality risk: the Framingham Heart Study. Arterioscler Thromb Vasc Biol 27:127-133

34. Lee DH, Lee IK, Porta M, Steffes M, Jacobs DR Jr (2007) Relationship between serum concentrations of persistent organic pollutants and the prevalence of metabolic syndrome among non- 
diabetic adults: results from the National Health and Nutrition Examination Survey 1999-2002. Diabetologia 50:1841-1851

35. Lee DH, Lee IK, Jin SH, Steffes M, Jacobs DR Jr (2006) Association between serum concentrations of persistent organic pollutants and insulin resistance among nondiabetic adults: results from the National Health and Nutrition Examination Survey 1999-2002. Diabetes Care 30:622-628

36. Ha MH, Lee DH, Jacobs DR Jr (2007) Association between serum concentrations of persistent organic pollutants and self-reported cardiovascular disease prevalence: results from the National Health and Nutrition Examination Survey 1999-2002. Environ Health Perspect 115:1204-1209

37. Lowell BB, Shulman GI (2005) Mitochondrial dysfunction and type 2 diabetes. Science 307:384-387

38. Pardini RS, Heidker JC, Baker TA, Payne B (1980) Toxicology of various pesticides and their decomposition products on mitochondrial electron transport. Arch Environ Contam Toxicol 9:87-97

39. Pardini RS (1971) Polychlorinated biphenyls (PCB): effect on mitochondrial enzyme systems. Bull Environ Contam Toxicol 6:539-545

40. Irigaray P, Mejean L, Laurent F (2005) Behaviour of dioxin in pig adipocytes. Food Chem Toxicol 43:457-460

41. Brewster DW, Matsumura F (1988) Reduction of adipose tissue lipoprotein lipase activity as a result of in vivo administration of 2,3,7,8-tetrachlorodibenzo- $p$-dioxin to the guinea pig. Biochem Pharmacol 37:2247-2253
42. Allsop M, Gohnston P (2000) Unseen poisons in Asia. A review of persistent organic pollutant levels in South and Southeast Asia and Oceania. Greenpeace Report. Available from http://www. greenpeace.to/publications/asiapops.pdf, accessed 16 November 2007

43. Dougherty CP, Henricks Holtz S, Reinert JC, Panyacosit L, Axelrad DA, Woodruff TJ (2000) Dietary exposures to food contaminants across the United States. Environ Res 84:170-185

44. Bayen S, Barlow P, Lee HK, Obbard JP (2005) Effect of cooking on the loss of persistent organic pollutants from salmon. J Toxicol Environ Health Part A 68:253-265

45. Joslin EP (1933) Fat and the diabetic. N Engl J Med 209:519-528

46. Pebble WE (1923) Obesity: observations on one thousand cases. Boston Med Surg J 188:617-621

47. Cleverly D, Schaum J, Winters D, Schweer G, O'Rourke K (1998) The inventory of sources of dioxin in the United States. Organohalogen Compounds 36:1-6

48. Needham LL, Barr DB, Caudill SP et al (2005) Concentrations of environmental chemicals associated with neurodevelopmental effects in U.S. population. Neurotoxicology 26:531-545

49. Luross JM, Alaee M, Sergeant DB et al (2002) Spatial distribution of polybrominated diphenyl ethers and polybrominated biphenyls in lake trout from the Laurentian Great Lakes. Chemosphere 46: $665-672$

50. Anway MD, Cupp AS, Uzumcu M, Skinner MK (2005) Epigenetic transgenerational actions of endocrine disruptors and male fertility. Science 308:1466-1469 